\title{
SÍNTESIS VERDE DE NANOPARTÍCULAS DE PLATA USANDO EL EXTRACTO ACUOSO DE LAS HOJAS DE AJO (Allium sativum)
}

\author{
GREEN SYNTHESIS OF SILVER NANOPARTICLES \\ USING THE AQUEOUS EXTRACT OF THE LEAVES \\ OF GARLIC PLANT (Allium sativum)
}

Fernanda Pilaquinga F. ${ }^{*}$, Katherine Pazmiño V. ${ }^{1}$, Alexandra Robalino T. ${ }^{1}$, Eliza Jara N. ${ }^{1}$, Fernanda López F. ${ }^{2}$, Lorena Meneses O. ${ }^{1}$, Karla Vizuete A. ${ }^{3}$ \& Alexis Debut M. ${ }^{3}$

Recibido: 30 de abril 2019 / Aceptado: 6 de junio 2019

DOI: 10.26807/ia.v7i2.102

Palabras clave: Allium sativum, ajo, hojas, nanopartículas de plata Keywords: Allium sativum, garlic, leaves, silver nanoparticles

\section{RESUMEN}

Se han descrito muchos estudios de biosíntesis de nanopartículas de plata usando el extracto del bulbo de ajo (Allium sativum) como agente reductor. Sin embargo, el uso de sus hojas es poco conocido, por considerarse un producto

1 Pontificia Universidad Católica del Ecuador, Facultad de Ciencias Exactas y Naturales, Escuela de Ciencias Químicas, Quito, Ecuador (*correspondencia: mfpilaquingaf@puce.edu.ec; katherine.pazmino@yahoo.es; alex-robalino@hotmail.com; enjara@puce.edu.ec; Immeneses@puce.edu.ec ).

2 Pontificia Universidad Católica del Ecuador-Sede Ibarra, Herbario, Ibarra, Ecuador (mflopez2@pucesi.edu.ec).

3 Universidad de las Fuerzas Armadas ESPE, CENCINAT, Sangolquí, Ecuador (ksvizuete@espe.edu.ec; apdebut@espe.edu.ec ). 
de desecho. En este estudio, se comparó el tamaño de nanopartículas de plata obtenidas con el extracto acuoso de las hojas de ajo usando dos técnicas diferentes de calentamiento: placa y por microondas. Las nanopartículas obtenidas se caracterizaron por espectrofotometría UV-Visible, microscopía de transmisión electrónica (TEM, por sus siglas en inglés), dispersión dinámica de luz (DLS, por sus siglas en inglés) y difracción de rayos X (XRD, por sus siglas en inglés). Como resultado se obtuvieron nanopartículas con un tamaño promedio

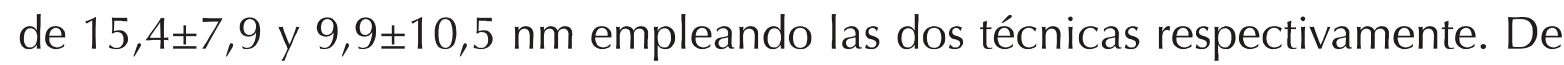
acuerdo al análisis estadístico realizado, se demostró que existen diferencias significativas en relación al tamaño de nanopartícula obtenido. Se concluye que el extracto acuoso de las hojas de la planta de ajo, es un agente reductor adecuado para la síntesis de nanopartículas de plata, y que la técnica de microondas es más efectiva por la rapidez y tamaño de nanopartículas obtenidas.

\section{ABSTRACT}

Many studies of biosynthesis of silver nanoparticles have been described using garlic bulb extract (Allium sativum) as a reducing agent. However, the use of its leaves is little known as it is considered a waste product. In this study, silver nanoparticles size obtained with the aqueous extract of the garlic leaves were compared using two different heating techniques: plate and microwave. The nanoparticles obtained were characterized by Visible UV spectrophotometry, electron transmission microscopy (TEM), dynamic light scattering (DLS) and Xray diffraction (XRD). As a result, the average size nanoparticles obtained were $15.4 \pm 7.9$ and $9.9 \pm 10.5 \mathrm{~nm}$, using both techniques. Based on the statistical analysis carried out it was proven that there are significant differences in relation to the size of the nanoparticle obtained. It is concluded that the aqueous extract of the leaves of garlic plant is a suitable reducing agent for the synthesis of silver nanoparticles and that the microwave technique is more effective because of the speed and size of nanoparticles obtained. 


\section{INTRODUCCIÓN}

La nanotecnología cada día se convierte en uno de los campos más investigados, debido al gran número de aplicaciones que proporciona en el área de la química, la biología, la medicina, la electrónica, entre otros. Las nanopartículas, con un tamaño menor a $100 \mathrm{~nm}$, pueden prepararse a través de diferentes técnicas, por medio de métodos químicos, electroquímicos, de radiación, fotoquímicos y biológicos. El método biológico realizado a través de la "síntesis verde", ha ganado notoriedad, ya que evita el uso de compuestos tóxicos y peligrosos. De igual manera disminuye el riesgo biológico que conlleva la experimentación con cultivos microbiológicos que requieren procedimientos complejos (Mittal et al., 2013; Roy \& Das, 2015).

El estudio de la síntesis verde durante los últimos 30 años ha permitido descubrir el potencial de los extractos de plantas para reducir iones metálicos, lo que resulta en la formación de nanopartículas de tamaños y morfologías estables. Se sugiere que los extractos de plantas cumplen con dos funciones: reducir y estabilizar a las nanopartículas. Además, de ser un método simple, económico y amigable con el medio ambiente (Mittal et al., 2013).

Las nanopartículas que contienen metales tales como la plata (AgNPs), son ampliamente utilizadas por su eficiente actividad óptica y antimicrobiana. Su efecto a nivel nanométrico está ligado a la alta relación entre su volumen y el área de superficie. Las nanopartículas de plata pueden ser fácilmente preparadas con extractos de plantas (Roy \& Das, 2015).

Uno de los principales campos de aplicación de las AgNPs preparadas con extractos de plantas, es la biomedicina, pues por su biocompatibilidad y baja toxicidad, pueden ser empleadas en el diagnóstico y terapias contra el cáncer. Así lo han reportado investigaciones que emplearon AgNPs con extractos, y como resultado, inhibieron el crecimiento de células cancerígenas de colon (Prabhu et al., 2013), y cuello uterino (HeLa) (Singh et al., 2018 \& Tripathi et al. 2019) 
Allium sativum, comúnmente conocida como ajo, forma parte de la familia Amaryllidaceae. Es una especie herbácea perenne, cuyo tallo puede Ilegar a medir 0,9 m. Está formada por un bulbo y hojas presentes al final del tallo. El ajo tiene un alto contenido de compuestos con azufre, que contribuyen a sus propiedades bioactivas. El bulbo de ajo, usado como medicina y condimento desde tiempos remotos, ha sido estudiado extensamente por sus propiedades laxante, diurética, antioxidante y anticancerígena; y actividades antimicrobiana, antifúngica, antiviral y antiprotozoaria. Además, su aplicación para el tratamiento de enfermedades causadas por agentes patógenos es muy amplia (Harris et al., 2001; Meriga et al, 2012).

Uno de los primeros estudios empleando bulbos de ajo, logró producir AgNPs altamente estables y con un gran potencial antibacteriano frente a microorganismos Gram positivos y Gram negativos (Rastogi \& Arunachalam, 2011). Estudios similares demostraron la biocompatibilidad del extracto de ajo con nanopartículas de oro (muy parecidas químicamente a la plata), empleadas como agentes de liberación de fármacos (Rastogi \&
Arunachalam, 2013). Por otro lado, al utilizar el extracto de ajo con otro tipo de nanopartículas como óxido de zinc, se demostró su capacidad bactericida frente a las cepas de Staphylococcus aureus, Bacillus subtilis, Listeria monocytogenes, Escherichia coli, Salmonella typhimurium y Pseudomonas aeruginosa (Stan et al., 2016).

Las propiedades ligadas a la composición química de las hojas de ajo son de gran interés dentro del campo de la química aplicada, sin embargo, no se han encontrado publicaciones que describan la síntesis de AgNPs con su extracto acuoso. Los únicos reportes encontrados hasta la fecha están relacionados al campo de la biología y biotecnología. Se ha estudiado la actividad biológica de flavonoides y otros compuestos en ensayos in vivo (Abdo \& Al-Kafawi, 1969) e in vitro (Broadhurst et al., 2000; Kim et al., 2005). Otros estudios, reportan las interacciones de la aglutinina presente en sus hojas, con otros grupos funcionales aislados de microorganismos para usarla como una proteína antifúngica (Banerjee et al., 2004; Ghosh et al., 2013). Por otro lado, otros grupos experimentaron con las 
lectinas de las hojas, aislando, caracterizando y clonándolas molecularmente (Smeets et al., 1997) para crear una proteína insecticida contra insectos homópteros presentes en plantas de tabaco transgénico (Dutta et al., 2005).
Con estos antecedentes, en este estudio se empleó el extracto acuoso de hojas de ajo (A. sativum) para producir nanopartículas de plata, empleando calentamiento por placa y microondas.

\section{MATERIALES Y MÉTODOS}

\section{Muestreo}

Hojas frescas de A. sativum se recolectaron en el sector de Quiroga, Ibarra-Ecuador (Figura 1), coordenadas geográficas: $0^{\circ} 17^{\prime} 11.3^{\prime \prime} \mathrm{N}, 78^{\circ} 17^{\prime} 14.5^{\prime \prime} \mathrm{O}$ a 2480 m.s.n.m.

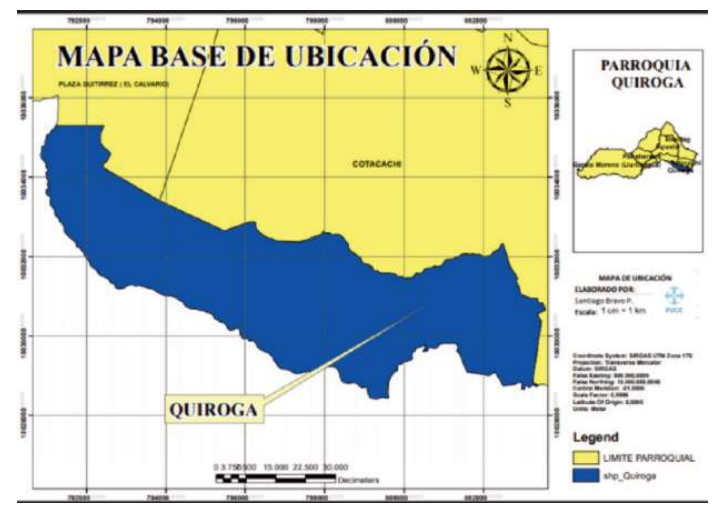

Figura 1. Sitio de muestreo de las hojas de A. sativum

Las muestras se desinfectaron con etanol al $60 \%$. Luego pasaron por un período de cuarentena a una temperatura de -2 a $-4{ }^{\circ} \mathrm{C}$ en un refrigerador marca Ecasa ${ }^{\circledR}$. Las hojas se secaron a una temperatura de $40{ }^{\circ} \mathrm{C}$ por 10 horas en un deshidratador marca Termokool ${ }^{\circledR}$, de acuerdo a lo sugerido por Morejón et al. (2018). Finalmente, las hojas se trituraron en un mortero de cuarzo.

\section{Preparación del extracto}

Para el extracto acuoso de las hojas de ajo, se empleó $1 \mathrm{~g}$ de muestra, 20 $\mathrm{mL}$ de agua destilada y calentamiento con agitación constante en una placa de calentamiento marca Velp ${ }^{\circledR}$ a $50{ }^{\circ} \mathrm{C}$ durante $1 \mathrm{~h}$. 


\section{Síntesis de AgNPs}

Para la síntesis de nanopartículas de plata se siguió el método sugerido por Kesharwani et al. (2009), con ligeras modificaciones. Se emplearon $20 \mathrm{~mL}$ de $\mathrm{AgNO}_{3}$ marca Scharlau $\mathbb{R}$ $1 \mathrm{mM}$ y $1 \mathrm{~mL}$ del extracto acuoso de hojas de ajo. Se ajustó el pH a 8 con $\mathrm{NaOH}$ marca Merck® al $1 \%$, y se mantuvo en agitación constante en la placa de calentamiento a 300 r.p.m. durante $1 \mathrm{~h}$ a $50{ }^{\circ} \mathrm{C}$ de temperatura. En el segundo método, se emplearon $20 \mathrm{~mL}$ de $\mathrm{AgNO}_{3} 1 \mathrm{mM}, 1 \mathrm{~mL}$ del extracto acuoso, a pH 8, tiempo 1 min, y potencia $800 \mathrm{~W}$ usando un microondas Indurama $\mathbb{R}$ modelo MWI 28BL.

\section{Caracterización de AgNPs}

Para caracterizar a las nanopartículas de plata obtenidas, se empleó un espectrofotómetro UV-Visible de doble haz Cary 60 Agilent Technologies ${ }^{\circledR}$ y celdas de cuarzo. Para el estudio de microscopía electrónica de transmisión (TEM) se usó un equipo de marca FEI®, modelo Tecnai Spirit G2. El radio hidrodinámico de las nanopartículas fue medido con un medidor de dispersión dinámica de luz (DLS) usando un Nanoparticle Size Analyzer LB-550 marca Horiba®. La presencia de plata en las muestras se analizó con un difractómetro de rayos $X$ (PANalytical) en configuración de Bragg-Brentano a 40 kV y 45 $\mathrm{mA}$ con un monocromador de rayos X de Cu K-alpha $(\square=1.541 \AA$ ).

\section{Tratamiento de datos}

Para analizar si existe diferencia significativa en el tamaño de nanopartícula obtenido con los tratamientos de calentamiento por placa y microondas, se emplearon pruebas t y $\mathrm{F}$ mediante Microsoft Excel. Previo al análisis de resultados, se eliminaron los valores atípicos mediante la evaluación de los rangos intercuartílicos (IQR score).

\section{RESULTADOS}

Los espectros UV-Vis de las AgNps usando placa de calentamiento (a) y microondas (b), en comparación con el extracto (c), se aprecian en la Figura 2. Las longitudes de onda máxima se obtuvieron a $435 \mathrm{~nm}$ para el 
caso del calentamiento con placa, $433 \mathrm{~nm}$ para el microondas y $319 \mathrm{~nm}$ para el extracto.

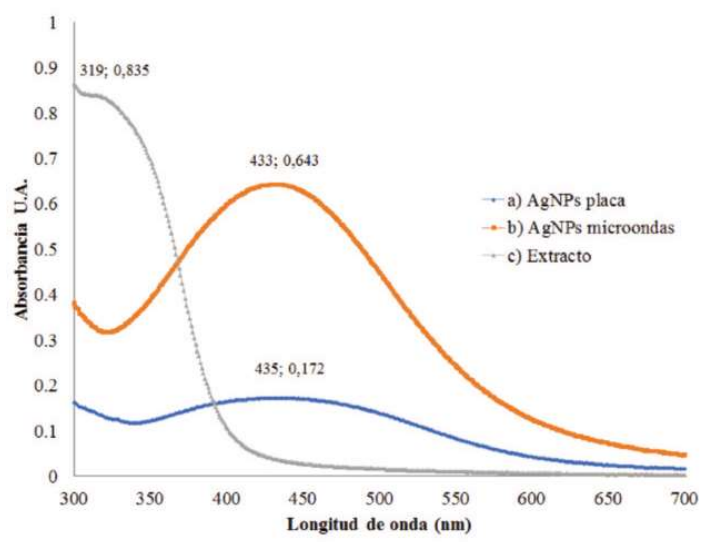

Figura 2. Espectros UV-Vis de AgNps usando extracto acuoso de hojas de ajo empleando (a) placa de calentamiento, (b) microondas de A. sativum (c) extracto de A. sativum

En la Figura 3 se muestran las micrografías TEM de las AgNPs, empleando las dos técnicas de calentamiento por placa (a) y microondas (b). Los histogramas de tamaño de nanopartícula se muestran en la Figura 4. El tamaño promedio fue de $15,4 \pm 7,9$ (4a) y 9,9 $\pm 10,5 \mathrm{~nm}(4 \mathrm{~b})$ respectivamente.

El tamaño del recubrimiento orgánico medido por el DLS, fue de 40,5 $\pm 8,3$ y $35,1 \pm 9,7 \mathrm{~nm}$ usando el extracto acuoso de hojas de ajo por calentamiento en placa y microondas, respectivamente. a)
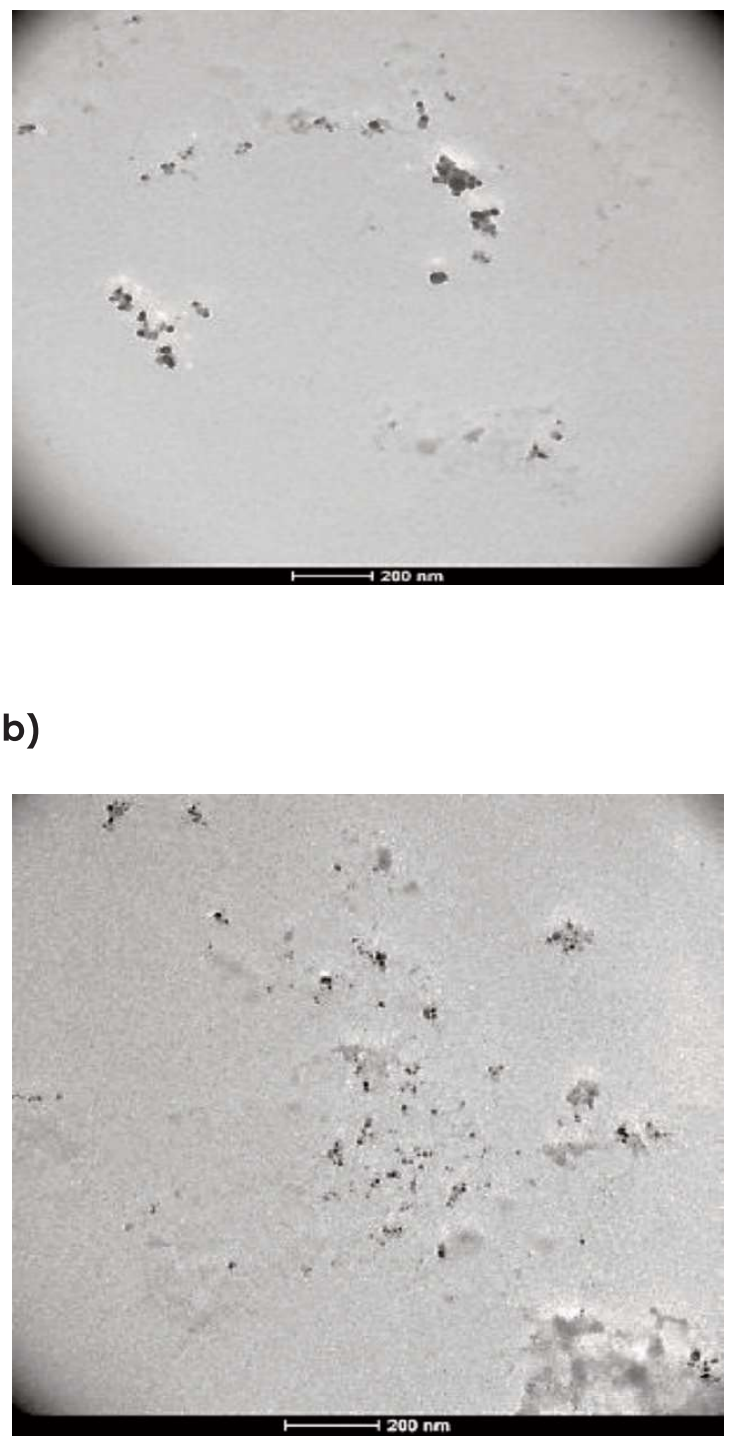

Figura 3. Micrografías TEM de AgNps usando extracto acuoso de hojas de ajo empleando (a) placa de calentamiento y (b) microondas 
a)

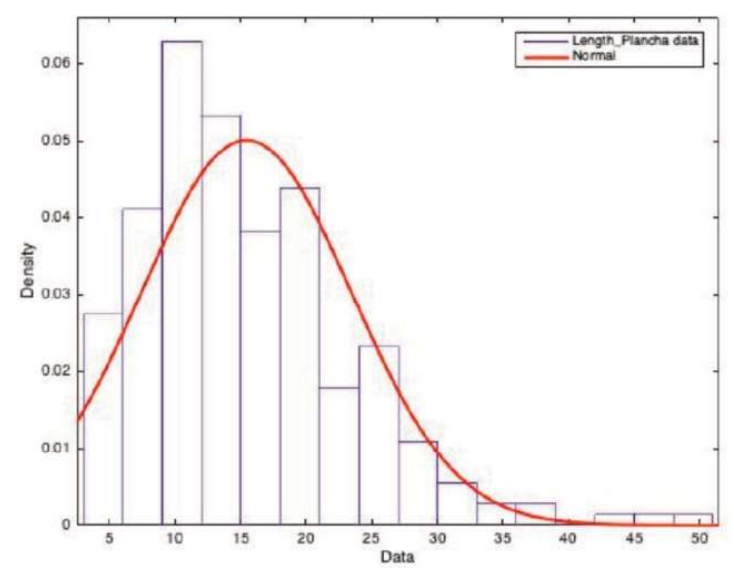

b)

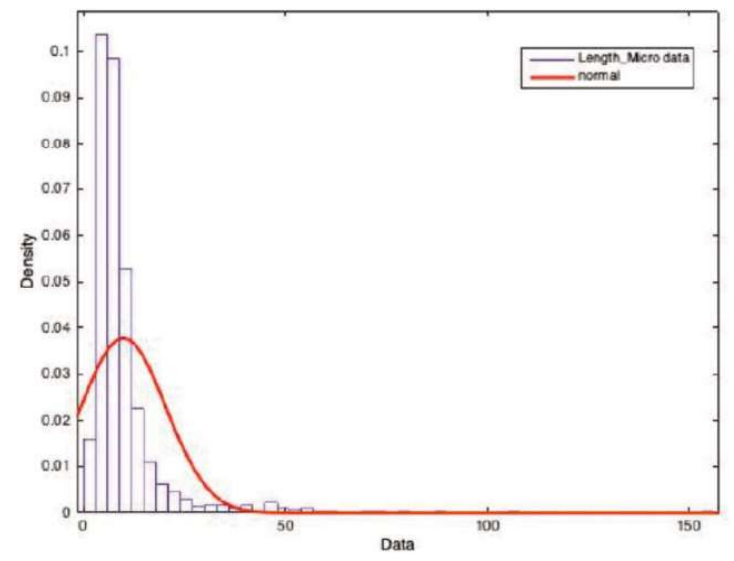

Figura 4. Histogramas de frecuencia de tamaño de AgNps usando extracto acuoso de hojas de ajo empleando

(a) placa de calentamiento y (b) microondas

En cuanto a la composición cristalina analizada por XRD (Figura 5), las dos muestras presentaron en el difractograma Ag en el ángulo $38,0^{\circ}$ y $\mathrm{NaCl}$ en placa de calentamiento (a): $27,8^{\circ} 28,4^{\circ} 32,3^{\circ} 35,9^{\circ}$; y microondas (b): $27,7^{\circ} 27,84^{\circ} 32,3^{\circ} 37,9^{\circ}$. (a

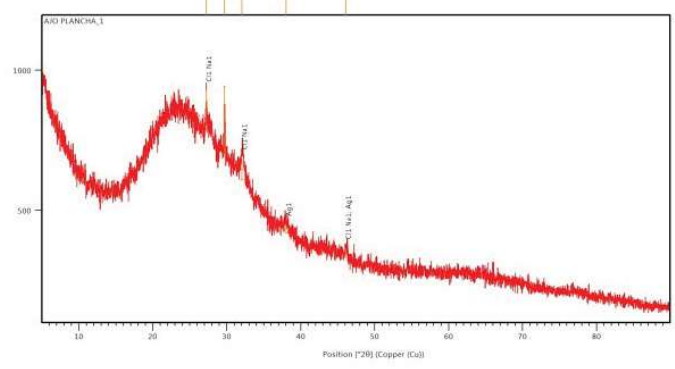

(b)

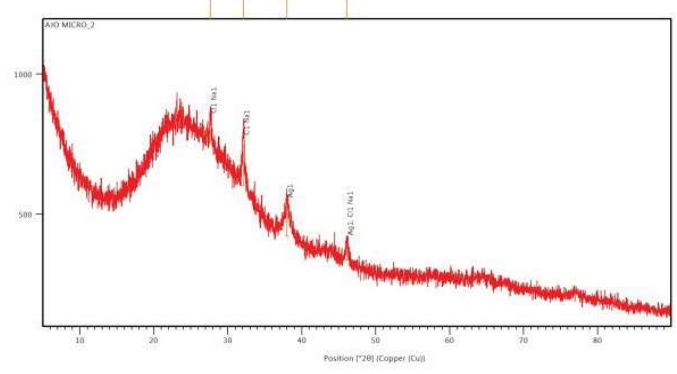

Figura 5. Difractogramas XRD de AgNps (a) placa y (b) microondas

Con los datos de las mediciones del diámetro de AgNPs, se realizaron pruebas de rango intercuartílico, que se muestran en la Figura 6, para cada tratamiento, con la finalidad de evaluar la presencia de valores atípicos. En el tratamiento con microondas se obtuvo un $7,9 \%$ de las mediciones identificadas como outliers, frente al $2,5 \%$ del otro tratamiento. Como se puede observar, se visualizan estos valores como los puntos fuera de la caja. Cabe señalar que en ambos casos se realizaron más de 100 mediciones. 


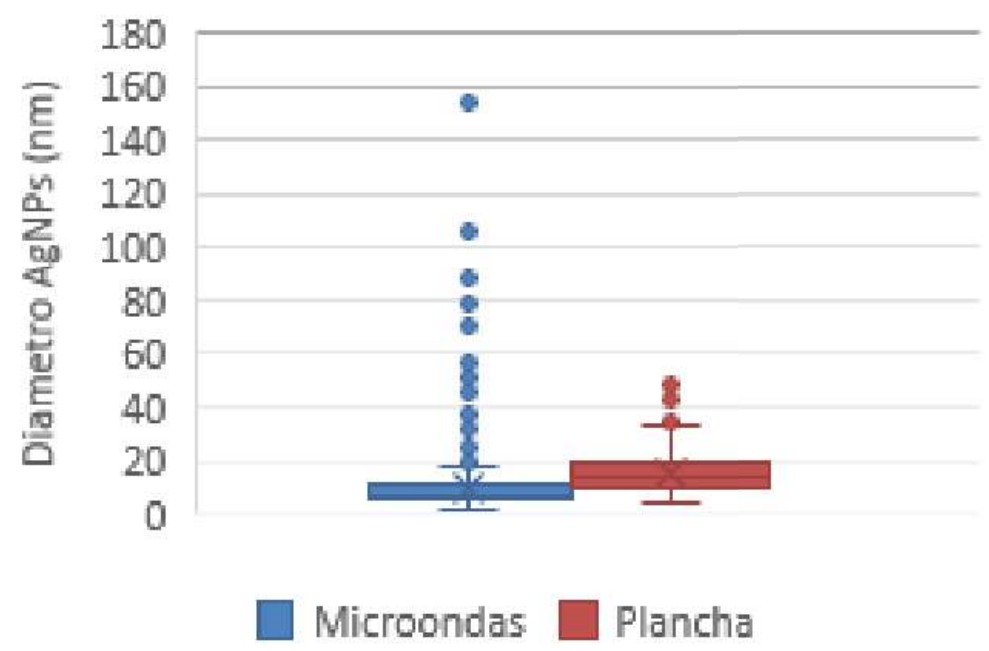

Figura 6. Diagramas de caja y bigote para la distribución del tamaño de AgNPs obtenidas con extracto de hojas de ajo y con cada tratamiento de calentamiento

Una vez identificados y eliminados los valores atípicos, se comparó si existe diferencia significativa en el tamaño de nanopartícula (prueba t) y su variabilidad (prueba F) con relación al tipo de calentamiento utilizado en el proceso de síntesis. Estos resultados se indican en la Tabla 1.

Tabla 1. Análisis estadístico de resultados del tamaño de AgNPs obtenidas con extracto de hojas de ajo y con cada tratamiento de calentamiento

\begin{tabular}{lcc}
\hline & Microondas & Placa \\
\hline Media (nm) & 7,56 & 14,78 \\
Varianza & 12,18 & 47,22 \\
$\mathrm{~N}$ & 950 & 238 \\
\hline \multicolumn{3}{c}{ Prueba t } \\
\hline \multicolumn{2}{c}{ Valor de p (2 colas) } \\
\hline \multicolumn{3}{c}{ Prueba F } \\
\hline \multicolumn{2}{c}{ Valor de $\mathrm{P}$} & 0 \\
\hline
\end{tabular}




\section{DISCUSIÓN}

Las AgNPs obtenidas mostraron un máximo de absorción a 435 nm usando placa de calentamiento y 433 $\mathrm{nm}$ con microondas, a diferencia del extracto, que muestra su absorción a los $319 \mathrm{~nm}$. De acuerdo a la estimación del tamaño de nanopartícula por Espectrofometría UV-Vis propuesta por Pradeep (2012), el plasmón de superficie de las AgNps se muestra a los $400 \mathrm{~nm}$, sugiriendo un valor aproximado con recubriendo orgánico de 30 a $50 \mathrm{~nm}$. El tamaño real medido por TEM, indicó que el menor valor fue el obtenido por medio de microondas $(9,9 \pm 10,5 \mathrm{~nm})$. La diferencia con el valor estimado por espectrofotometría UV-Vis, se debe a que la micrografía TEM muestra únicamente el núcleo metálico de Ag y no el recubrimiento orgánico. La micrografía de la Figura $2 \mathrm{~b}$ usando microondas, además, muestra un gran número de nanopartículas esféricas, a diferencia de la muestra obtenida por placa de calentamiento.

De acuerdo a la composición química de las plantas, existen diferentes grupos funcionales como los azúcares reductores, compuestos fenólicos o ácidos orgánicos, que facilitan la reducción de iones metálicos (Ahmed et al., 2016; Mittal et al., 2013). El ajo contiene compuestos orgánicos que actúan como agentes reductores y estabilizantes (Bender-Bojalil \& Bárcenas-Pozos, 2013). Al comparar el tamaño de AgNps usando el extracto de hojas con el de bulbo de ajo, los resultados de este estudio muestran tamaños mucho menores que los reportados en la bibliografía (Cardeño, 2014).

Las medidas realizadas por medio de DLS indican el espesor de la capa de recubrimiento del material orgánico para los dos tipos de calentamiento (Ramesh et al., 2016). El menor tamaño de nanopartícula recubierta se dio usando microondas. Los valores de DLS obtenidos concuerdan con el tamaño de nanopartícula estimado por espectrofotometría UV-Vis (30 a $50 \mathrm{~nm}$ ).

El análisis cristalográfico confirmó la presencia Ag en el ángulo $38,0^{\circ}$, correspondiente al plano (111) de una estructura cúbica (Vizuete et al., 2016). La mayor cantidad de Ag se 
encontró en las muestras preparadas por microondas, lo cual significa que este proceso es más eficiente en la generación de nanopartículas.

De acuerdo con el análisis estadístico, existe diferencia significativa entre el tamaño de nanopartícula obtenido con placa de calentamiento y microondas. Los resultados de la Tabla 1 indican que en ambos casos se rechaza la hipótesis nula por haberse obtenido un valor $\mathrm{p}$ menor a 0,05, por lo tanto, el tratamiento de calentamiento tiene una influencia significativa en el resultado. Con la síntesis por microondas se obtuvieron AgNPs de menor tamaño $(9,9 \mathrm{~nm})$ y con una distribución más homogénea, ya que, al eliminar los valores atípicos, el $40 \%$ de las mediciones se encuentran en el rango $\pm 25 \%$ de la media, esto comparado a las nanopartículas obtenidas con placa de calentamiento, cuyo tamaño promedio fue de 15,4 nm y donde sólo el $8 \%$ de los valores se encuentran en el rango $\pm 25 \%$ de la media, lo que denota una mayor dispersión de los diámetros obtenidos.

Se sugiere la síntesis vía microondas, debido a su rapidez y cantidad de nanopartículas obtenidas (Ahmed et al., 2016; Mittal et al., 2013; Von White et al., 2012). Estudios similares, avalan el uso de microondas en la síntesis de nanopartículas de plata empleando extractos de plantas (Kahrilas et al., 2014).

\section{CONCLUSIÓN}

El extracto acuoso de las hojas de ajo A. sativum es un agente reductor adecuado para la formación de AgNps, por su potencial aplicación en el campo biomédico. Los tamaños promedio obtenidos fueron de 15,4 $\pm 7,9$ y 9,9 $\pm 10,5 \mathrm{~nm}$ usando dos técnicas de calentamiento (placa y microondas) respectivamente. Los tamaños hidrodinámicos fueron de 40,5 $\pm 8,3$ y 35,1 $\pm 9,7$ nm. El análisis cristalográfico confirmó la presencia de plata en las muestras analizadas. El tratamiento estadístico del tamaño de nanopartículas obtenidas, indica que existe una diferencia significativa de acuerdo al tipo de calentamiento, por lo que se sugiere el uso del microondas para la síntesis de AgNPs. 


\section{AGRADECIMIENTO}

Este estudio fue realizado dentro del proyecto PUCE "Síntesis, caracterización y modelamiento computacional de nanopartículas de plata usando extractos de plantas nativas de la provincia de Imbabura", Código O13083, QINV0080-IINV529020200.

\section{LISTA DE REFERENCIAS}

Abdo, M. S., \& Al-Kafawi, A. A. (1969). Biological Activities of Allium Sativum. The Japanese Journal of Pharmacology, 19(1), 1-4.

Ahmed, S., Ahmad, M., Swami, B. L., \& Ikram, S. (2016). A review on plants extract mediated synthesis of silver nanoparticles for antimicrobial applications: A green expertise. Journal of Advanced Research, 7(1), 17-28.

Bender-Bojalil, D., \& Bárcenas-Pozos, M. E. (2013). El ajo y sus aplicaciones en la conservación de alimentos. Temas Selectos de Ingeniería de Alimentos, 7(1), 25-36.

Broadhurst, C. L., Polansky, M. M., \& Anderson, R. A. (2000). Insulin-like Biological Activity of Culinary and Medicinal Plant Aqueous Extracts in Vitro. Journal of Agricultural and Food Chemistry, 48(3), 849-852.

Cardeño, L., \& Londoño, M. E. (2014). Síntesis verde de nanopartículas de plata mediante el uso del ajo (Allium sativum). Revista Soluciones de Postgrado EIA, 6(12), 129-140.

Dutta, I., Saha, P., Majumder, P., Sarkar, A., Chakraborti, D., Banerjee, S., \& Das, S. (2005). The efficacy of a novel insecticidal protein, Allium sativum leaf lectin (ASAL), against homopteran insects monitored in transgenic tobacco. Plant Biotechnology Journal, 3(6), 601-611. 
Ghosh, P., Roy, A., Chakraborty, J., \& Das, S. (2013). Biological Safety Assessment of Mutant Variant of Allium sativum Leaf Agglutinin (mASAL), a Novel Antifungal Protein for Future Transgenic Application. Journal of Agricultural and Food Chemistry, 61(48), 11858-11864.

Harris, J. C., Cottrell, S. L., Plummer, S., \& Lloyd, D. (2001). Antimicrobial properties of Allium sativum (garlic). Applied Microbiology and Biotechnology, 57(3), 282-286.

Kahrilas, G. A., Wally, L. M., Fredrick, S. J., Hiskey, M., Prieto, A. L., \& Owens, J. E. (2014). Microwave-Assisted Green Synthesis of Silver Nanoparticles Using Orange Peel Extract. ACS Sustainable Chemistry \& Engineering, 2(3), 367-376.

Kesharwani, J., Yoon, K. Y., Hwang, J., \& Rai, M. (2009). Phytofabrication of Silver Nanoparticles by Leaf Extract of Datura metel: Hypothetical Mechanism Involved in Synthesis. Journal of Bionanoscience, 3(1), 39-44.

Kim, M.-Y., Kim, Y.-C., \& Chung, S.-K. (2005). Identification and in vitro biological activities of flavonols in garlic leaf and shoot: inhibition of soybean lipoxygenase and hyaluronidase activities and scavenging of free radicals. Journal of the Science of Food and Agriculture, 85(4), 633-640.

Meriga, B., Mopuri, R., \& MuraliKrishna, T. (2012). Insecticidal, antimicrobial and antioxidant activities of bulb extracts of Allium sativum. Asian Pacific Journal of Tropical Medicine, 5(5), 391-395.

Mittal, A. K., Chisti, Y., \& Banerjee, U. C. (2013). Synthesis of metallic nanoparticles using plant extracts. Biotechnology Advances, 31(2), 346-356.

Morejón, B., Pilaquinga, F., Domenech, F., Ganchala, D., Debut, A., \& Neira, M. (2018). Larvicidal Activity of Silver Nanoparticles Synthesized Using Extracts of Ambrosia arborescens (Asteraceae) to Control Aedes aegypti L. (Diptera: Culicidae). Journal of Nanotechnology, 2018, 1-8.

Prabhu, D., Arulvasu, C., Babu, G., Manikandan, R., \& Srinivasan, P. (2013). Biologically synthesized green silver nanoparticles from leaf extract of Vitex negundo $L$. induce growth-inhibitory effect on human colon cancer cell line HCT15. Process Biochemistry, 48(2), 317-324. 
Pradeep, T. (2012). A Textbook of Nanoscience and Nanotechnology (1 Ed.). Nueva Deli: McGraw Hill.

Ramesh, S., Grijalva, M., Debut, A., de la Torre, B. G., Albericio, F., \& Cumbal, L. H. (2016). Peptides conjugated to silver nanoparticles in biomedicine - a "valueadded" phenomenon. Biomaterials Science, 4(12), 1713-1725.

Rastogi, L., \& Arunachalam, J. (2011). Sunlight based irradiation strategy for rapid green synthesis of highly stable silver nanoparticles using aqueous garlic (Allium sativum) extract and their antibacterial potential. Materials Chemistry and Physics, 129(12), 558-563.

Rastogi, L., \& Arunachalam, J. (2013). Green synthesis route for the size controlled synthesis of biocompatible gold nanoparticles using aqueous extract of garlic (Allium sativum). Advanced Materials Letters, 4(7), 548-555.

Roy, S., \& Das, T. K. (2015). Plant Mediated Green Synthesis of Silver Nanoparticles-A Review. International Journal of Plant Biology \& Research, 3(03), 1044-1055.

Singh, H., Du, J., Singh, P., \& Yi, T. H. (2018). Ecofriendly synthesis of silver and gold nanoparticles by Euphrasia officinalis leaf extract and its biomedical applications. Artificial Cells, Nanomedicine, and Biotechnology, 46(6), 1163-1170.

Smeets, K., Van Damme, E. J. M., Verhaert, P., Barre, A., Rougé, P., Van Leuven, F., \& Peumans, W. J. (1997). Isolation, characterization and molecular cloning of the mannose-binding lectins from leaves and roots of garlic (Allium sativum L.). Plant Molecular Biology, 33(2), 223-234.

Stan, M., Popa, A., Toloman, D., Silipas, T.-D., \& Vodnar, D. C. (2016). Antibacterial and Antioxidant Activities of ZnO Nanoparticles Synthesized Using Extracts of Allium sativum, Rosmarinus officinalis and Ocimum basilicum. Acta Metallurgica Sinica (English Letters), 29(3), 228-236.

Tripathi, D., Modi, A., Narayan, G., \& Rai, S. P. (2019). Green and cost effective synthesis of silver nanoparticles from endangered medicinal plant Withania coagulans and their potential biomedical properties. Materials Science and Engineering: C, 100, 152-164. 
Vizuete, K. S., Kumar, B., Vaca, A. V., Debut, A., \& Cumbal, L. (2016). Mortiño (Vaccinium floribundum Kunth) berry assisted green synthesis and photocatalytic performance of Silver-Graphene nanocomposite. Journal of Photochemistry and Photobiology A: Chemistry, 329, 273-279.

Von White, G., Kerscher, P., Brown, R. M., Morella, J. D., McAllister, W., Dean, D., \& Kitchens, C. L. (2012). Green synthesis of robust, biocompatible silver nanoparticles using garlic extract. Journal of Nanomaterials, 2012, 55. 